\title{
Quantification of the antibody response to Propionibacterium acnes in a patient with prosthetic valve endocarditis: - a case report
}

\author{
T. Herren ${ }^{1 *}$, M. A. Middendorp ${ }^{2}$ and R. Zbinden ${ }^{3}$
}

\begin{abstract}
Background: The isolation of Propionibacterium acnes in blood cultures is often considered a contaminant. On rare occasions, $P$. acnes can cause severe infections, including endocarditis and intravascular prosthesis-associated infections. To evaluate the discrimination between a contaminant and a clinically relevant infection we used an Ouchterlony test system to quantify the antibody response to $P$. acnes in a patient with a proven $P$. acnes endocarditis.

Case presentation: We report on a 64-year-old Caucasian man who developed $P$. acnes endocarditis four years following a composite valve-graft conduit replacement of the aortic root. Bacterial growth in blood cultures was detected after an incubation period of 6 days. However, the antibody titer to $P$. acnes was 1:8 at the time of diagnosis and declined slowly thereafter over $2 \frac{1}{2}$ years. The patient's response to the antibiotic treatment was excellent, and no surgical re-intervention was necessary.

Conclusion: The working hypothesis of infective endocarditis can be substantiated by serologic testing, which, if positive, provides one additional minor criterion. Moreover, quantification of the antibody response to $P$. acnes, though not specific, may assist in the differentiation between contaminants and an infection. This quantification may have implications for the patient management, e.g. indication for and choice of the antibiotic therapy.
\end{abstract}

Keywords: Infective endocarditis, Prosthetic heart valve, Propionibacterium acnes, Serologic tests, Bacteremia, Contamination

\section{Background}

Propionibacterium acnes is often considered a contaminant when it grows in blood culture, akin to the growth of coagulase-negative staphylococci, Bacillus sp., and Corynebacterium sp. In a series of 522 patients with $P$. acnes bacteremia, only 18 (3.5\%) were considered to have a clinically significant bacteremia [1]. Diagnosis of the latter required at least two sets of blood cultures showing growth of $P$. acnes, and the patients had to have a systemic inflammatory response syndrome [1]. In equivocal cases, the availability of a serologic test for $P$.

\footnotetext{
* Correspondence: thomas.herren@spital-limmattal.ch

${ }^{1}$ Department of Medicine, Limmattal Hospital, Urdorferstrasse 100, CH-8952

Schlieren, Switzerland

Full list of author information is available at the end of the article
}

acnes would assist in the differentiation between true infection and contaminant bacterial growth. Moreover, the slow growth of $P$. acnes in blood cultures requires prolonged incubation periods of up to two weeks [2]. In patients with suspected infective endocarditis, antibiotic therapy often cannot be withheld for such a long duration. A higher diagnostic precision is desired in such circumstances, and a positive serologic test for $P$. acnes would confirm the diagnosis. Furthermore, the concentration time course of the antibody titers can show a decline in case of the elimination of $P$. acnes, or a late rise indicating a relapse. Antibodies against $P$. acnes were characterized using enzyme-linked immunosorbent assays (ELISAs) in patients with acne vulgaris [3] and in patients with prostatitis and benign prostatic hyperplasia 
[4]. To our knowledge, serologic testing in patients with suspected $P$. acnes endocarditis has not been reported before. Thus, we describe a concentration time graph of the antibody titer to $P$. acnes in a patient with proven infective endocarditis. The potential clinical application of serologic testing is discussed, and specific features of $P$. acnes endocarditis, especially regarding its sometimes difficult diagnosis, are outlined.

\section{Case presentation}

A 64-year-old Caucasian man had severe regurgitation of the tricuspid aortic valve due to an aneurysm of the ascending aorta, which involved the sinus of Valsalva. Composite valve-graft conduit replacement of the aortic root was performed 4 years ago. The patient suffered a transient ischemic attack with a thrombotic occlusion of a branch of the left middle cerebral artery 18 months postoperatively, most likely due to subtherapeutic oral anticoagulation (international normalized ratio [INR] 1.8). Transesophageal echocardiography (TEE) did not show vegetations on the mechanical aortic valve and the concentration of C-reactive protein was $1 \mathrm{mg} / \mathrm{L}$ (normal value $<5)$. Aspirin was then added to the vitamin $\mathrm{K}$ antagonist phenprocoumon, and the patient successfully self-monitored his INR values with a target range of 2.0-3.0. He did not use skin disinfectants, and he had no acne.

A wasp sting to his upper lip was managed conservatively one month prior to hospitalization, and subdued sounds of his mechanical aortic valve were noticed by the patient. No dental procedures were performed in the six months prior to the sting. He became febrile up to $38{ }^{\circ} \mathrm{C}$, had night sweats and felt ill. Upon admission, the patient was in good general condition. His body temperature was $37.3{ }^{\circ} \mathrm{C}$; his blood pressure was $137 /$ $94 \mathrm{mmHg}$; and his pulse rate was $79 \mathrm{bpm}$. There was a $1 / 6$ systolic murmur and a metallic hue to the second heart sound. There were no peripheral emboli in the skin. The spleen was not enlarged. Laboratory results showed an elevated concentration of C-reactive protein (73 mg/L, Fig. 1) and a normal leukocyte count $\left(7.3 \times 10^{9} / \mathrm{L}\right.$ [normal values $\left.\left.4.0-9.8\right]\right)$. The kidney function (creatinine $90 \mu \mathrm{mol} / \mathrm{L}[62-110]$ ) and the liver function tests (aspartate transaminase $[\mathrm{AST}] 29 \mathrm{U} / \mathrm{L}[<38]$ ) were normal. The INR value was 2.4 , consistent with therapeutic anticoagulation with phenprocoumon. The lactic dehydrogenase was slightly elevated (655 U/L $[<248])$. An infective endocarditis was suspected clinically, and TEE was performed. Vegetations on the mechanical bileaflet aortic valve were seen (Fig. 2), but there was no perivalvular abscess formation. Ten blood cultures (Bactec $^{\mathrm{TM}}$ 9050, Becton Dickinson, Franklin Lakes, NJ) were inoculated with $10 \mathrm{ml}$ blood samples, five cultured under aerobic and five under anaerobic conditions. All
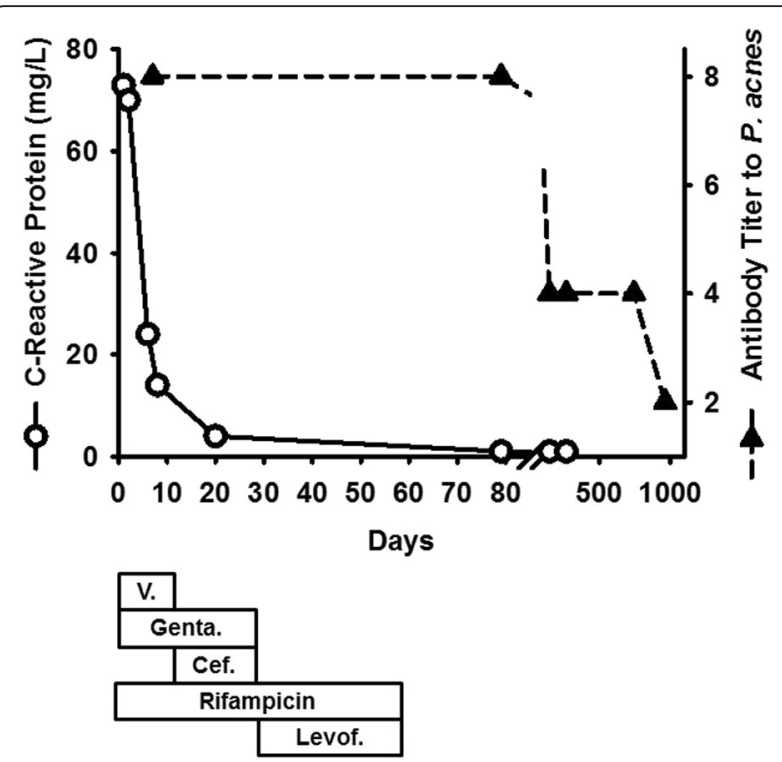

Fig. 1 Concentration time graph of the C-reactive protein (open circles, solid line) and the anti - P. acnes antibody titer (filled triangles, dashed line). The latter was measured using the Ouchterlony immuno-diffusion test. The antibiotic therapy is shown in the horizontal bars below the graph $(\mathrm{V}$. = Vancomycin, Genta. = Gentamicin, Cef. = Ceftriaxone, Levof. = Levofloxacin)

blood cultures were obtained before starting the antibiotic therapy. Initially, no bacterial growth was observed. Broad range polymerase chain reaction (PCR) using $16 \mathrm{~S}$ rDNA primers failed to identify bacteria in blood samples. One anaerobic blood-culture showed growth of a Gram-positive rod after 6 days, which was sent to the reference laboratory for further testing. The identification of $P$. acnes was performed according to standard procedures with conventional tests. The Grampositive rods were catalase positive, showed a positive

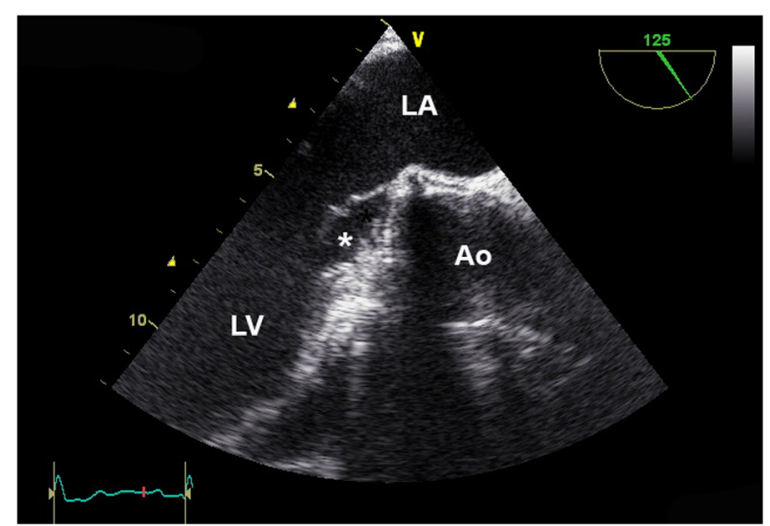

Fig. 2 Photograph obtained during transesophageal echocardiography (long axis view of the left ventricular outflow tract). Two vegetations $\left(^{*}\right.$ ) are visible on the mechanical bileaflet aortic valve, protruding into the left ventricular outflow tract in diastole. Ao = aorta, LA = left atrium, $\mathrm{LV}=$ left ventricle 
CAMP reaction, reduced nitrate and produced indole, but were lipase and lecithinase negative. Utilizing gasliquid chromatography, the production of propionic acid from the glucose broth was detected. In four of 5 anaerobic blood cultures bacteria grew within 12 days, and biochemical analysis again identified $P$. acnes; all aerobic blood cultures remained sterile. Furthermore, the antibody response to this bacterium was quantified (Fig. 1). According to the modified Duke criteria, a definitive diagnosis of infective endocarditis was confirmed [5]. The initial antibiotic treatment included vancomycin and gentamicin i.v. plus rifampicin p.o. After the identification of the $P$. acnes, vancomycin was stopped and ceftriaxone was started. The intravenous therapy was stopped after four weeks, and levofloxacine plus rifampicin were prescribed for an additional four weeks of oral antibiotic therapy. No bacteria grew in the blood cultures following the completion of the antibiotic therapy. A follow-up TEE showed no vegetations on the mechanical aortic valve, and the patient continues to be well. $\mathrm{He}$ consistently uses skin disinfectants prior to obtaining a blood specimen for his INR measurements.

Sera drawn at different time points were tested for antibodies against $P$. acnes by the Ouchterlony assay [6] in the following $2 \frac{1}{2}$ years. For the antigen preparation of the $P$. acnes strain (ATCC 6916, Manassas, VA) colonies of 20 sheep blood agar plates were suspended in a tube with $10 \mathrm{ml}$ of phosphate buffered saline $(\mathrm{pH} 7.2)$ and sonicated in a cup horn sonifier (60 W, Branson Sonic Power Co., Danbury, CT) 8 times for one minute. The tube was centrifuged at 2000xg for $10 \mathrm{~min}$ following overnight storage at $4{ }^{\circ} \mathrm{C}$. Aliquots of the supernatant, each containing $100 \mu \mathrm{L}$, were stored at $-80{ }^{\circ} \mathrm{C}$. The patient's sera were tested against this antigen with the Ouchterlony test [7]. The highest dilution of the serum that still precipitated the $P$. acnes antigen in the Ouchterlony test system (ID Plates Cleargel, IMMY, Norman, $\mathrm{OK})$ represented the titer of this serum. The titers decreased during the following $2 \frac{1}{2}$ years indicating the clearing of the bacteria without relapse (Fig. 1).

\section{Discussion}

Propionibacterium spp. are Gram-positive, slow-growing anaerobic, non-spore-forming rods. They are part of the normal flora of the skin, nasopharynx, oral cavity, and genitourinary tract. Generally thought to be nonpathogenic in humans, they can cause infective endocarditis, especially in carriers of mechanical heart valves and pacemakers or implantable cardioverter defibrillators (ICDs) [8]. This may be due to their ability to adhere to foreign body surfaces and produce a biofilm. In a series of 58 patients with infective endocarditis due to Propionibacterium spp. published in 2009, prosthetic valve endocarditis occurred in $67 \%$ of the patients and was the most common presentation of Propionibacterium spp. infection. In addition to antimicrobial treatment, $81 \%$ of the patient underwent cardiac surgery (due to the propensity of the organism to form an abscess [9]), and the overall mortality rate was $16 \%[8]$. The patient described above was cured with antibiotic therapy alone. Most of the infections of mechanical heart valves occur at the time of surgery. With high inocula, a short incubation period is expected. However, $P$. acnes may reside intracellularly in macrophages and remain there dormant for years [10]. In our patient, the infection may also have occurred during the frequent skin punctures necessary for the determination of the level of anticoagulation [11]. Propionibacterium endocarditis may be difficult to diagnose. The median incubation period required for blood cultures to become positive is 7 days (range 5-14 days) [2]. Therefore, a prolonged incubation period of two weeks is proposed in cases of suspected infection with Propionibacterium spp. Additional diagnostic methods are often necessary. PCR to detect $16 \mathrm{~S}$ rDNA in blood is used in cases of blood culturenegative infective endocarditis. However, its sensitivity is lower than that of blood culture methods, and blood specimens need to be pretreated to remove background bacterial DNA contamination [12]. Gas-liquid chromatographic examination of subcultured bacterial colonies may assist in the detection of anaerobic bacteria [13]. In blood culture-negative infective endocarditis, which occurs in $2.5-31 \%$ of all cases of infective endocarditis, serologic techniques may be useful [14], and are established to diagnose infections including Brucella spp., Coxiella burnetii [5], Bartonella spp., Mycoplasma spp., and Legionella spp.[15]. In the patient described above, an antibody response to $P$. acnes was quantified (Fig. 1), showing a titer of $1: 8$ at the time of diagnosis and a gradual decline thereafter. Therefore, this antibody could potentially facilitate the early diagnosis of infective endocarditis due to $P$. acnes. When uncertainty exists regarding whether the growth of $P$. acnes in blood cultures is a contaminant, as occurs in $>95 \%$ of cases [1], or whether it has a true pathogenetic role, a detectable antibody titer would support the latter. However, increased antibody titers against $P$. acnes have been reported in diseases such as acne vulgaris [16] or benign prostatic hyperplasia [4], lowering their specificity. In patients with acne a time course of the serology can detect a rising antibody titer suggesting $P$. acnes infection.

Treatment of $P$. acnes infective endocarditis primarily consists of a $\beta$-lactam antibiotic, often combined with an aminoglycoside [8], even though P. acnes is frequently resistant to the latter [17]. Oral rifampicin must be added because of its ability to penetrate the bacterial biofilm [8] and may be combined with a chinolone antibiotic [18]. In vitro data suggest that the combination of 
daptomycin with rifampicin is highly active against $P$. acnes biofilms [19]. Long-term antibiotic therapy is required for the successful treatment of infectious endocarditis due to $P$. acnes. The treatment effect is mirrored by the decreasing antibody titer.

\section{Conclusions}

1. In patients with suspected $P$. acnes infective endocarditis, a high antibody titer to the bacterium is useful diagnostic adjunct. According to the modified Duke criteria, serological evidence of active infection represents a minor criterion for the diagnosis of infective endocarditis [5].

2. Serologic testing may be useful in cases of $P$. acnes bacteremia to differentiate between blood culture contamination and a clinically relevant infection.

3. The Ouchterlony test used in the present case report allows the analysis of the antibody titer to $P$. acnes over time. Therefore, it may be useful even in acne patients, even though the specificity will be lower. Validation in a larger clinical cohort including blood donors and patients with acne as controls is important.

\section{Ethics and consent to publish}

The patient was identified during routine clinical practice and consented to give venous blood samples after elaborate information. Involvement of the ethical committee of the Canton of Zurich was not considered necessary, since the project was not based on a study protocol, and is not classified as research by the Swiss Federal Act on Research on Human Beings. Written informed consent was obtained from the patient for publication of this case report and all accompanying images. A copy of the written consent is available for review by the editor of this journal.

\section{Availability of supporting data}

The data set supporting the results of this article is depicted in Fig. 1. P. acnes used for the Ouchterlony test system was from the American Type Culture Collection (ATCC 6916), headquartered in Manassas, VA.

\section{Abbreviations \\ AST: aspartate transaminase; CAMP: Christie Atkins Munch-Petersen; ELISA: enzyme-linked immunosorbent assay; ICD: internal cardioverter defibrillator; INR: international normalized ratio; PCR: polymerase chain reaction; rDNA: ribosomal deoxyribonucleic acid; TEE: transesophageal echocardiography.}

\section{Competing interests}

The authors declare that they have no competing interests.

\section{Authors' contributions}

TH was the staff physician in charge of the patient, and performed the transesophageal echocardiography, created the graphs, and drafted the manuscript. MAM carried out the serologic testing and critically reviewed the manuscript. RZ conceived of the case report, devised the Ouchterlony test, and assisted in drafting the manuscript. All authors read and approved the final manuscript.

\section{Authors' information}

RZ is a Professor of Microbiology at the University of Zurich in Switzerland. $\mathrm{He}$ is a M.D. and holds a master's degree in science. He heads the clinical microbiology and molecular diagnostics unit of the Institute for Medical Microbiology at the University of Zurich, and is a board member of the Association of the Swiss Medical Laboratories.

\section{Acknowledgment}

We are indebted to Dr. Gerhard Eich, M.D., infectious disease consultant at the Triemli Hospital in Zurich, Switzerland, for his expert advice on the choice of the antibiotic treatment.

Funding

Article-processing charges were defrayed in part by the Forschungsfonds of the Spital Limmattal.

\section{Author details}

'Department of Medicine, Limmattal Hospital, Urdorferstrasse 100, CH-8952 Schlieren, Switzerland. ²Department of Surgery, Kantonsspital, Im Ergel 1, $\mathrm{CH}-5404$ Baden, Switzerland. ${ }^{3}$ Microbiological Laboratory, Limmattal Hospital, Urdorferstrasse 100, CH-8952 Schlieren, Switzerland.

Received: 22 July 2015 Accepted: 20 April 2016

Published online: 29 April 2016

\section{References}

1. Park HJ, Na S, Park SY, Moon SM, Cho OH, Park KH, et al. Clinical significance of Propionibacterium acnes recovered from blood cultures: analysis of 524 episodes. J Clin Microbiol. 2011;49(4):1598-601. doi:10.1128/JCM.01842-10.

2. Clayton JJ, Baig W, Reynolds GW, Sandoe JA. Endocarditis caused by Propionibacterium species: a report of three cases and a review of clinical features and diagnostic difficulties. J Med Microbiol. 2006;55(Pt 8):981-7. doi:10.1099/jmm.0.46613-0.

3. Burkhart CG, Cantrill J, Butcher CL, Lehmann PF. Propionibacterium acnes: interaction with complement and development of an enzyme-linked immunoassay for the detection of antibody. Int J Dermatol. 1999; 38(3):200-3.

4. Shannon BA, Cohen RJ, Garrett KL. The antibody response to Propionibacterium acnes is an independent predictor of serum prostatespecific antigen levels in biopsy-negative men. BJU Int. 2008;101(4):42935. doi:10.1111/j.1464-410X.2007.07214.x.

5. Li JS, Sexton DJ, Mick N, Nettles R, Fowler Jr VG, Ryan T, et al. Proposed modifications to the Duke criteria for the diagnosis of infective endocarditis. Clin Infect Dis. 2000;30(4):633-8.

6. Ouchterlony $\mathrm{O}$. Antigen-antibody reactions in gels. IV. Types of reactions in coordinated systems of diffusion. Acta Pathol Microbiol Scand. 1953;32(2):230-40.

7. Middendorp MA. Nachweis von Antikörper gegen Propionibacterium acnes bei Patienten mit pulmonaler Hypertonie und bei Patienten mit Endocarditis. Zurich: University of Zurich; 2015

8. Sohail MR, Gray AL, Baddour LM, Tleyjeh IM, Virk A. Infective endocarditis due to Propionibacterium species. Clin Microbiol Infect. 2009;15(4):387-94. doi:10.1111/j.1469-0691.2009.02703.x.

9. List RJ, Sheikh N, Theologou T, Mitchell IM, Mathew T. Propionibacterium acnes endocarditis of a prosthetic aortic valve. Clin Cardiol. 2009;32(8):E46-7. doi:10.1002/clc.20492.

10. Jakab E, Zbinden R, Gubler J, Ruef C, von Graevenitz A, Krause M. Severe infections caused by Propionibacterium acnes: an underestimated pathogen in late postoperative infections. Yale J Biol Med. 1996;69(6):477-82.

11. O'Neill TM, Hone R, Blake S. Prosthetic valve endocarditis caused by Propionibacterium acnes. Br Med J (Clin Res Ed). 1988;296(6634):1444.

12. Madico GE, Rice PA. 16S-Ribosomal DNA to diagnose culture-negative endocarditis. Curr Infect Dis Rep. 2008;10(4):280-6.

13. Phillips KD, Tearle PV, Willis AT. Rapid diagnosis of anaerobic infections by gas-liquid chromatography of clinical material. J Clin Pathol. 1976;29(5):428-32. 
14. Zbinden R, Hany A, Luthy R, Conen D, Heinzer I. Antibody response in six HACEK endocarditis cases under therapy. APMIS. 1998;106(5):547-52.

15. Authors/Task Force M, Habib G, Lancellotti P, Antunes MJ, Bongiorni MG, Casalta JP, et al. ESC Guidelines for the management of infective endocarditis: The Task Force for the Management of Infective Endocarditis of the European Society of Cardiology (ESC)Endorsed by: European Association for Cardio-Thoracic Surgery (EACTS), the European Association of Nuclear Medicine (EANM). Eur Heart J. 2015;36(44):3075-128. doi:10.1093/ eurheartj/ehv319.

16. Basal E, Jain A, Kaushal GP. Antibody response to crude cell lysate of Propionibacterium acnes and induction of pro-inflammatory cytokines in patients with acne and normal healthy subjects. J Microbiol. 2004; 42(2):117-25.

17. Wang WL, Everett ED, Johnson M, Dean E. Susceptibility of Propionibacterium acnes to seventeen antibiotics. Antimicrob Agents Chemother. 1977;11(1):171-3.

18. Mory F, Fougnot S, Rabaud C, Schuhmacher H, Lozniewski A. In vitro activities of cefotaxime, vancomycin, quinupristin/dalfopristin, linezolid and other antibiotics alone and in combination against Propionibacterium acnes isolates from central nervous system infections. J Antimicrob Chemother. 2005;55(2):265-8. doi:10.1093/jac/dkh521.

19. Furustrand Tafin U, Corvec S, Betrisey B, Zimmerli W, Trampuz A. Role of rifampin against Propionibacterium acnes biofilm in vitro and in an experimental foreign-body infection model. Antimicrob Agents Chemother. 2012;56(4):1885-91. doi:10.1128/AAC.05552-11.

\section{Submit your next manuscript to BioMed Central and we will help you at every step:}

- We accept pre-submission inquiries

- Our selector tool helps you to find the most relevant journal

- We provide round the clock customer support

- Convenient online submission

- Thorough peer review

- Inclusion in PubMed and all major indexing services

- Maximum visibility for your research

Submit your manuscript at www.biomedcentral.com/submit

) Biomed Central 